\title{
Echanges globaux, agents locaux. l'apport de la capoeira aux études sur la mondialisation
}

Daniel Granada (UNIVATES)
Cet article ${ }^{1}$ interroge l'apport des concepts d'immigration, globalisation, diaspora et transnationalisation pour comprendre le processus d'expansion hors du Brésil de la capoeira, un art martial d'origine " afro-brésilienne " très pratiquée dans plusieurs pays du monde. La recherche de terrain a été réalisée en France et au Royaume-Uni entre 2007 et 2011, par le biais de l'observation participante, des interviews informelles et plus de soixante-dix interviews formelles enregistrées avec les capoeiristas de différents groupes de Paris et sa banlieue et Londres. La recherche visait à appréhender les transformations opérées par les capoeiristas sur la pratique de la capoeira dans ce processus de délocalisation de la pratique brésilienne et sa conséquente réappropriation par des pratiquants en Europe. Une partie de cet effort se traduit par la nécessité de trouver un cadre théorique permettant de comprendre les limitations de l'application conceptuelle au cas concret. Le présent article explore les limites et potentialités des différents outils conceptuels dans la compréhension du processus d'expansion de la capoeira pour ensuite proposer l'interprétation de ce phénomène en trois moments distincts mais reliés entre eux, sans être rattachés à une temporalité strictement déterminée.

\section{COMMENT COMPRENDRE LA CAPOEIRA HORS DU BRESIL?}

II n'est pas surprenant que les premiers à avoir signalé la croissance de la capoeira hors du Brésil soient ceux ayant étudié l'émigration des Brésiliens vers les ÉtatsUnis (cf. Margolis 1994; Ribeiro 1999; Penha 2001; Martes 2003). Un grand nombre de Brésiliens qui ont émigré en quête de meilleures conditions de vie et de travail entre les années 1980 et les années 2000 ont emporté avec eux la pratique de la capoeira. Ils ont vu en cette pratique un moyen de survivre tout en restant en contact avec le Brésil.

L'émigration des Brésiliens aux États-Unis est un mouvement migratoire caractérisé, entre autres, par l'importance accordée aux réseaux sociaux tissés par les Brésiliens dans I'explication de ce phénomène (Martes 1999; Margolis 2003; 
Patarra 2005). Une recherche réalisée à Boston en 1995 démontre que les Brésiliens qui optent pour émigrer vers les États-Unis partent en quête d'opportunités de travail et principalement de l'ascension sociale qui leur a été refusée au Brésil (Sales 1999: 33). L'émigration des Brésiliens vers les États-Unis serait une immigration de « classe moyenne ", car elle rend nécessaire certaines ressources qui garantissent l'achat du billet d'avion (Sales 2005).

La crise économique des années 1980 au Brésil, appelée la " décennie perdue ", est vue comme le jalon décisif qui a déclenché l'émigration des Brésiliens. Sales (1999: 28) ajoute le facteur politique associé principalement aux espoirs et désenchantements de la période de redémocratisation du pays traversé par l'échec des divers plans économiques, l'augmentation du chômage et de l'inflation. Patarra (2005) explore l'hétérogénéité des mouvements migratoires à partir du Brésil et vers le Brésil, et signale l'existence de divers phénomènes et de divers groupes sociaux qui immigrent. L'auteur remarque la nécessité de prendre en compte dans l'explication des phénomènes migratoires du Brésil contemporain l'importance de la circularité de ces mouvements ainsi que les réseaux sociaux qui se constituent et se renforcent (Patarra 2005: 25-26).

Dans les études sur les Brésiliens aux USA, la capoeira, la samba et la batucada sont souvent présentées comme des manifestations de "brésilité " ou "afro-brésilité " ${ }^{2}$. Cependant, quelques études ont conclu que la participation des Brésiliens au sein de ces groupes n'était pas très représentative en nombre d'adhérents (Margolis 1994:307). Les groupes de capoeira et de batucada sont, hors du Brésil, des groupes d'expression de la "brésilité " qui pour la plupart ne sont pas intégrés par des Brésiliens mais par la population locale. En général, seuls les enseignants et un petit nombre restreint de Brésiliens font partie de ces groupes, malgré la grande présence d'immigrés provenant du Brésil aux États-Unis ${ }^{3}$.

Dans une étude de référence sur les immigrants brésiliens à New York, Margolis (1994) note l'absence de "liens communautaires " au sein de la " communauté des Brésiliens " de New York ${ }^{4}$. Cette constatation a été faite lors de l'observation des activités d'un centre de promotion de la " culture brésilienne " à New York:

Um outro exemplo da ausência de entrosamento comunitário é a falta de apoio para as aulas de especialidades tradicionalmente brasileiras tais como a "capoeira" e a "batucada", oferecidas por aquele centro. Estas aulas, e outras similares oferecidas de forma particular, atraíram apenas alguns entre os milhares de brasileiros que residem na cidade de Nova York. Por exemplo, entre as dezenas de alunos das aulas de "capoeira " oferecidas em Nova York, desde meados dos anos 1970, apenas cinco dos matriculados são atualmente brasileiros. Entre os frequentadores das escolas de samba locais, 90 por cento não eram brasileiros ; (...). Aparentemente, estes grupos são integrados por americanos aficionados da cultura e da música brasileiras, mais do que brasileiros envolvidos com sua herança cultural (Margolis 1994: 307).

Ce qui est interprété par Margolis comme un exemple d'absence de liens communautaires, ou un manque d'intérêt envers les spécialités brésiliennes, constitue en effet l'une des caractéristiques principales du processus d'implantation de la capoeira à l'étranger. Lors de ma recherche de terrain, la majeure partie des pratiquants de capoeira, dans les groupes que j'ai étudiés en France et au Royaume-Uni, ne sont pas brésiliens ${ }^{5}$. De façon générale, le nombre de Brésiliens n'est pas très important dans les groupes de capoeira hors du Brésil, même dans des villes où l'immigration brésilienne est considérable comme à Paris et à Londres. 
Après cette première vague d'études sur l'immigration des Brésiliens aux États-Unis, deux études ont été consacrées à l'analyse de la pratique de la capoeira et de son appropriation hors du Brésil: les études pionnières de Travassos (2000) sur la capoeira aux États-Unis et celles de Vassallo (2001) sur la capoeira en France.

Les théories sur les migrations internationales occupent une place centrale dans l' explication de l'expansion de la pratique de la capoeira hors du Brésil. Des travaux récents, comme celui d'Aceti (2011) sur l'expansion de la capoeira en Europe et la thèse de Guizardi (2011) qui traite de la capoeira en Espagne et à Madrid, montrent l'importance accordée aux théories sur l'immigration pour expliquer l'expansion de la capoeira en Europe 6 . Certes, la perspective des études sur les migrations internationales est importante pour expliquer les origines et la diffusion de la pratique de la capoeira en Europe. Néanmoins, la perspective des migrations internationales présente deux problèmes centraux. Premièrement, le cadre assimilationniste de ces études (Capone 2010: 236-237) est remis en cause puisque c'est justement par le biais de la valorisation positive dans l'espace public d'un « bien culturel » issu de son pays d'origine que le capoeirista réussit son intégration dans la société d'accueil. Deuxièmement, ce ne sont pas uniquement les immigrés brésiliens qui font partie des groupes de capoeira, puisque, comme nous l'avons vu, la plupart des pratiquants et membres des groupes de capoeira étudiés ne sont pas Brésiliens ${ }^{7}$. En général, seul le mestre ou le professeur, voire ponctuellement un autre élève, sont Brésiliens. Dans les groupes analysés dans ma thèse, trois leaders étaient des non-Brésiliens, tout comme la grande majorité des élèves à Paris et à Londres. Certains groupes étaient même composés exclusivement de pratiquants non-brésiliens ${ }^{8}$. Cela dit, il ne s'agit pas d'oblitérer l'apport des théories sur les migrations internationales dans l'explication du phénomène de l'expansion de la capoeira hors du Brésil, mais il est nécessaire d'exploiter d'autres perspectives qui aident à comprendre la délocalisation et la conséquente relocalisation de la capoeira hors du Brésil. Les débats sur la globalisation, la diaspora et la transnationalisation deviennent ainsi des outils précieux pour comprendre ce phénomène.

\section{LA "GLOBALISATION " DE LA CAPOEIRA}

L'utilisation du concept de globalisation permet de comprendre et de mettre en évidence certains aspects du processus d'expansion de la capoeira hors du Brésil ${ }^{9}$. En général, la globalisation est vue comme un processus dans lequel l'accélération des échanges internationaux gagne partout de l'ampleur, car elle est principalement associée à l'intégration de l'économie mondiale. Comme le rappelle Abélès (2008: 134), le terme évoque l'interdépendance accélérée, le rétrécissement du monde avec l'érosion des frontières géographiques aux activités socio-économiques, l'action à distance et la compression de l'espace et du temps. Pour l'auteur, son usage renvoie à une conception de la globalisation marquée par la magnitude et l'intensité des changements d'échelle et des échanges interrégionaux:

II est clair en tout cas que l'usage du concept de globalisation renvoie à une perception de la mondialisation qui met l'accent sur les changements d'échelle, la magnitude croissante, I'accélération, l'impact plus intense des flux et des modèles d'interaction sociale interrégionaux (Abélès 2008: 134). 
Pour Robertson, il est possible d'identifier cinq phases de ce processus de globalisation, qu'il cherche à classer dans une perspective historique. À partir d'une " phase embryonnaire " au XVème siècle, ce processus se prolongerait au XVIIlème siècle, en passant par trois autres phases, jusqu'à atteindre la période des années 1960, appelée par Robertson la " phase de l'incertitude ". Cette dernière est caractérisée, entre autres, par la consolidation des médias globaux et par la multiplication de problèmes associés à la multinationalité (Robertson 1992:27).

Dans le cas de la capoeira, Assunção (2005: 208) signale la complexité de l'application de ce concept. Nul doute que le processus récent des flux d'échanges et d'interactions au niveau international exerce un impact remarquable sur la capoeira au Brésil et sur son expansion vers d'autres pays. Toutefois, comme l'explique Assunção, la capoeira serait issue d'un processus encore plus complexe d'interaction sociale interrégionale:

The term globalization is commonly used to suggest that the process is a recent, late twentieth-century development. In fact, one can hardly imagine a more momentous process of dislocation of peoples and cultures than the one produced by the slave trade for almost four centuries. The difference is that it happened outside Europe in distant colonies; metropolitan cultures only superficially acknowledged the social and cultural impact of the slave trade. They were not directly involved in the way they are now, when the metropolises themselves have become multicultural societies. That is why metropolitan cultures now need diasporic forms such as capoeira, which have accumulated a long experience of how to accommodate cultural diversity whilst still preserving a core identity (Assunção 2005: 208).

La formation de la capoeira au Brésil serait traversée par cette idée, comme une pratique délocalisée de la terre originelle, relocalisée et récréée au Brésil, et à partir des années 1970, réappropriée et relocalisée de nouveau dans divers pays du monde. Quand nous pensons à la capoeira, nous sommes depuis toujours amenés à penser à la multi-localité afin d'appréhender cette pratique dans toute sa complexité, issue d'innombrables déplacements, rencontres et changements qui sont à la base de sa formation et de sa reproduction. Nous sommes aussi obligés de prendre en compte la capoeira dans toute sa diversité, car elle varie selon les différentes régions du Brésil, selon l'appropriation des groupes, et les rapports conflictuels entretenus par certains groupes et certaines modalités de capoeira, dans un contexte où ces divisions sont reproduites dans l'expansion de la pratique hors du Brésil (Assunção 2005: 201). Ces processus innovants de re-sémantisation de la capoeira peuvent dans certains cas être expliqués par la notion de glocalization, pour reprendre le terme consacré par Robertson (1992). Cette notion, qui signale l'impact du global sur le local et la "localisation " des contenus globaux, peut servir à mettre en lumière certains aspects des processus d'adaptation et resémantisation de la capoeira dans des groupes et des associations hors du Brésil. Cependant, elle ne permet pas d'expliquer les liens qu'entretiennent ces individus et ces groupes au-delà des frontières, dans un contexte où la pratique est devenue transnationale.

II serait alors approprié de réserver le concept de globalisation, dans le cas des études sur la capoeira, pour faire référence à des processus plus larges, au niveau des échanges et des flux entre les nations, associés à la circulation d'images de la capoeira diffusées par les médias, par l'internet ou par les films ${ }^{10}$. Ces appropriations, destinées à un public plus large, montrent des adaptations de la capoeira où les idées sur le Brésil et les Brésiliens sont manipulées et réinterprétées selon des représentations locales ${ }^{11}$. 


\section{L'USAGE DE LA NOTION DE "DIASPORA "ET LA CAPOEIRA}

D'autres auteurs choisissent d'utiliser le terme " diasporique " pour designer l'expansion récente de la capoeira hors du Brésil. Ce choix est justifié par le lien entre l'expansion de la capoeira et le nombre de Brésiliens qui émigrent. Les partisans de cette approche soutiennent que les cours de capoeira sont offerts par des Brésiliens expatriés et auto-exilés, et que ces " écoles " de capoeira sont toujours basées au Brésil (Delamont et Stephens 2008):

Capoeira teachers all over the world have a homeland and aspire to return to it. Our research is on a globalized phenomenon, but we use the term diasporic because the teachers are Brazilians, who express saudade (nostalgic, homesick longing) for Brazil, and present themselves to their students as self-exiled, nomadic Brazilians. The term diasporic is particularly apt for the African-Brazilian capoeira teachers (Delamont et Stephens 2008: 60).

Une analyse de l'usage de la notion de diaspora révèle cependant certaines difficultés d'emploi de ce terme dans le cas de la capoeira. Le mot grec qui donne origine au terme diaspora provient du verbe grec spirein (semer), et plus précisément de sa forme composée diaspirein (disséminer) (Chivallon 2006; Cohen 1997, 2008). Historiquement, le terme diaspora a été utilisé pour désigner la dispersion des Juifs, des Grecs et des Arméniens. Du fait de l'exil forcé de ces peuples et de l'hostilité avec laquelle ils étaient reçus dans les nouveaux pays, le terme diaspora avait une connotation négative exprimant la tristesse associée au destin incertain de ces peuples (Cohen 1997: ix $)^{12}$. Actuellement, le terme " diaspora " est employé pour désigner les groupes sociaux les plus divers, jusque-là désignés par les termes " expulsés, déplacés, expatriés, exilés, réfugiés, immigrés, minorités ", entre autres (Bordes-Benayoun et Schnapper 2006:11-12). Il serait possible d'y ajouter les groupes de capoeira hors du Brésil. Néanmoins, l'innombrable diversité d'utilisation de ce terme met en cause sa propre application, autrement dit, l'extension du concept le mettrait en cause au cas où il n'aurait pas été « vidé de toute véritable compréhension " (Benayoun et Schnapper 2006:12). Les applications les plus diverses de ce terme rendent incompatibles l'usage contemporain du terme avec son usage classique (Faist 2010:13) ${ }^{13}$.

Parler d'une " diaspora de Brésiliens " ou d'une "diaspora de capoeiristas ", ce qui serait encore plus problématique, pour comprendre l'expansion de la capoeira hors du Brésil n'apporterait pas d'éclaircissements à la comprehénsion du phénomène de son expansion puisqu'il s'agit là d'une notion trop imprécise. Si nous comprenons la diaspora à partir du modèle, élaboré par Cohen $(1997,2008)$, des caractéristiques communes aux différentes diasporas, le cas des Brésiliens et de la capoeira a bien des difficultés à s'y retrouver ${ }^{14}$. Même s'il est possible d'associer certains aspects de l'émigration des Brésiliens vers d'autres pays aux éléments qui caractérisent une diaspora, réduire l'expansion de la capoeira à une diaspora de Brésiliens ou de capoeiristas signifierait effacer une des caractéristiques centrales du phénomène d'expansion de la capoeira hors du Brésil, à savoir l'appropriation de la pratique par les pratiquants locaux non-brésiliens. Si dans la plupart des cas les mestres et les leaders des groupes sont Brésiliens, les élèves sont toujours dans leur grande majorité des non-Brésiliens.

À la différence des approches qui mettent en avant la notion de " diaspora ", la perspective développée au long de mon travail de thèse souligne le rôle central des non-Brésiliens depuis le début de l'expansion de la 
capoeira hors du Brésil. La participation des non-Brésiliens dans ce mouvement ne doit donc pas être sous-estimée. Fréquemment ce sont eux qui souhaitent adhérer au groupe des mestres ou qui les invitent à s'installer dans leurs pays afin de créer leurs groupes à l'étranger. Les pratiquants non-brésiliens ont une grande importance dans la propagation et la manutention du mouvement d'expansion de la capoeira ${ }^{15}$. À ces faits s'ajoute l'évidence, dans les dernières années, de l'existence de plus en plus de groupes de capoeira à Londres et à Paris qui sont dirigés ou composés, en partie ou exclusivement, par des non-Brésiliens, qui s'approprient la capoeira et la pratiquent en s'adaptant aux contextes locaux, comme cela a été constaté lors de la recherche de terrain et développé au long de la thèse ${ }^{16}$.

II ne s'agit pas de rejeter la notion de diaspora, puisque le débat autour de cette notion s'avère important dans la compréhension de certaines caractéristiques de l'expansion de la capoeira, telles que l'alliance avec les "locaux ", I'idéalisation du Brésil comme Terre mère et l'envie de rentrer dans le pays d'origine ${ }^{17}$. Dans l'expansion de la capoeira hors du Brésil, certains groupes spécifiques s'approprient la pratique, en se réclamant Afro-descendants et en exigeant la reconnaissance de leur spécificité comme partie d'un mouvement diasporique. Dans leurs discours, ils expriment une volonté commune d'adhérer à un mouvement diasporique plus large, en voulant s'affilier au groupe des "Afro-descendants ". II serait alors possible d'appréhender les capoeiristas qui s'identifient de cette manière en tant que membres d'un mouvement diasporique plus large, associé à des identifications et à des identités flexibles et malléables (Hall 1990: 222-223). Cela constitue un modèle que Chivallon (2002: 52) a appelé " diaspora hybride ", et qui met en avant le mouvement, l'interconnexion et la mixité des références. Ce modèle signale la mobilité que touche même les " constructions » identitaires des diaspora. Dans cette production, il n'y a pas de continuité, ni de tradition nécessaire, mais de nouvelles réorganisations et reconfigurations où les références à la nation et aux idéologies nationalistes sont rejetées (Bruneau 2010:37). Dans cette perspective, la notion de diaspora pour la capoeira pourrait être employée sans qu'il y ait de référence directe à des Brésiliens, mais en se tournant vers des liaisons avec un mouvement diasporique et identitaire afro-descendant, où des agents locaux ou des capoeiristas font la connexion entre la pratique relocalisée et ce qui peut être identifié en tant qu'« africain » dans chaque contexte culturel.

Certains capoeiristas établis à Paris et à Londres, Brésiliens ou non, se considèrent être des " gardiens de la tradition ", ils se voient participer à une " culture afro-brésilienne " et veulent recréer hors du Brésil un "AfroBrésil ", dans lequel eux-mêmes et la capoeira qu'ils pratiquent participent, de manière créative, à un même passé mythique réactualisé dans le nouveau contexte d'insertion:

(...) it may make sense to try and reserve the idea of tradition for the nameless, evasive, minimal qualities that make these diaspora conversations possible. This would involve keeping the term as a way to speak about the apparently magical processes of connectedness that arise as much from the transformation of Africa by diaspora cultures as from the affiliation of diaspora cultures to Africa and the traces of Africa that those diaspora cultures enclose (Gilroy 1993: 199). 
De cette manière ils établissent des liens d'affinité entre eux et les "Africains de la diaspora " par des connections imaginées, rendues possibles grâce aux caractéristiques imputées à la capoeira dans sa formation et expansion. Cette pratique est alors conçue en tant que pratique délocalisée d'Afrique et relocalisée au Brésil, issue d'un mélange de " cultures variées ". Ce sont sur ces caractéristiques minimales que va se construire l'identification. L'emploi de la notion de tradition dans ce travail se rapproche ainsi de la notion de " travelling cultures » de Clifford (1997) qui est particulièrement intéressante pour le cas de la capoeira ${ }^{18}$. Dans sa formation et son développement, cette pratique peut être comprise à travers les " narrations ", le " mouvement ", la " transformation ", les connexions et relations, au lieu de l'attachement aux racines ou des mouvements de retour aux sources.

La « tradition » dans la capoeira est ainsi appréhendée comme étant définie par des connexions d'appartenance fluide, plutôt que par des formes rigides de liaison à une tradition singulière: "Identifications not identities, acts of relationship rather than pregiven forms: this tradition is a network of partially connected histories, a persistently displaced and reinvented time/space of crossing" (Clifford 1997: 268) ${ }^{19}$.

L'usage des notions de "diaspora" et de "tradition" peut aussi nous aider à mettre en évidence certaines caractéristiques de l'expansion de la capoeira pratiquée hors du Brésil, principalement en ce qui concerne les réappropriations et les réélaborations de ce qui peut symboliquement signifier " l'Afrique " ou le " Brésil " au sein de ces groupes. Or, d'une manière générale, cette réélaboration a pour objectif de mieux réussir l'intégration de la capoeira sur le " marché des produits ethniques " déjà bien développé dans des villes comme Paris et Londres.

\section{LA TRANSNATIONALISATION DE LA CAPOEIRA}

La notion de transnationalisation s'avère d'importance centrale dans l'étude de la capoeira hors du Brésil, puisqu'il ne s'agit plus de comprendre l'expansion de la capoeira comme étant associée exclusivement à un mouvement migratoire, mais également à des réseaux complexes qui ont été tissés au cours des dernières années entre des Brésiliens et des non-Brésiliens, au Brésil et ailleurs. Cette notion a été utilisée dans les études traitant de la capoeira hors du Brésil pour désigner des phénomènes liés à l'immigration des Brésiliens et la conséquente circulation de biens et d'idées au travers des frontières nationales. Ces études mettent en évidence les questions des rapports entre le " culturel " et le " politique " au sein des divers contextes nationaux, aussi bien que les implications identitaires et les rapports de pouvoir issus de la rencontre entre les Brésiliens et les pratiquants locaux au Canada (Joseph 2008a e 2008b), en France (Granada 2005, 2008, 2010 ; 2015 ; Gravina 2010) et en Espagne (Guizardi 2011) ${ }^{20}$. Plus récemment, des études ont mis en lumière l'impact de la transnationalisation de cette pratique au Brésil, avec les exigences croissantes sur la légitimité et la professionnalisation des enseignants et mestres de capoeira (Wesolowski 2012).

Comme l'explique Capone (2010), c'est dans les années 1990 que l'approche transnationale commence à s'affirmer dans les études sur les migrations. Cette nouvelle façon d'aborder les études migratoires met en avant la perspective des migrants, à savoir une perspective "from below ", "depuis le bas ", où les réseaux familiaux et les liens d'amitié de ces immigrés gagnent en visibilité. Différemment de la perspective des migrations internationales 
qui met en avant les échanges entre les États-nations "le terme 'transnational' définit toute activité initiée et menée par des acteurs non-institutionnels, qu'ils soient des groupes organisés ou des individus qui traversent les frontières des États-nations " (Capone 2010:237). La notion de transnationalisation serait ainsi particulièrement répandue dans les études anthropologiques du fait de l'intérêt croissant des chercheurs envers les échanges et les flux de personnes et d'éléments culturels à travers les frontières (Basch et al. 1994:50).

Ce sont les études pionnières de Basch, Glick-Schiller et Szanton Blanc (1994: 50) qui ont marqué le surgissement de la notion de transnationalisation dans le champ d'étude des migrations transnationales, avec en outre l'élaboration du concept de transmigrant:

Transmigrants are immigrants whose daily lives depend on multiple and constant interconnections across international borders and whose public identities are configured in relationship to more than one nation-state. (...) However, at the very same time, they are engaged elsewhere in the sense that they maintain connections, build institutions, conduct transactions, and influence local and national events in the countries from which they emigrated (Basch et al. 1994: 48).

Dans cette perspective, les études sur la transnationalisation impliquent le questionnement de la prémisse néo-classique des études sur les migrations internationales qui stipule que l'immigré cherchera à s'intégrer dans la société d'accueil par le biais de la suppression de toute manifestation publique à caractère identitaire, en les reléguant à l'environnement privé de sa vie domestique. Au contraire, dans la perspective de ces auteurs, le transmigrant reconfigure son identité publique en rapport avec plus d'un État-nation, sans cacher cette double appartenance. Dans le cas des groupes de capoeira installés à Paris et à Londres cette double appartenance est notable dans les liaisons que certains capoeiristas vont maintenir avec les groupes au Brésil, avec des effets sur les deux pôles de la circulation.

Quelques années plus tard, Hannerz (1996) va proposer l'usage du terme " transnationalisation ", au lieu de " globalisation ", pour expliquer des phénomènes à l'échelle des individus et des groupes. Cette notion permet de mettre en évidence la variation des phénomènes et la caractéristique singulière qu'ils partagent, celle de ne pas avoir une appartenance exclusive à un seul État, comme l'explique l'auteur:

The term " transnational " is in a way more humble, and often a more adequate label for phenomena which can be of quite variable scale and distribution, even when they do share the characteristic of not being contained within a state. It also makes the point that many of the linkages in question are not "international", in the strict sense of involving nations -actually, states - as corporate actors. In the transnational arena, the actors may now be individuals, groups, movements, business enterprises, and in no small part it is this diversity of organization that we need to consider (Hannerz 1996: 6).

Si l'usage du terme " transnationalisation " a trouvé une importante acceptation dans les milieux académiques, l'appropriation et l'usage équivoque du concept ont été récemment critiqués par Glick-Schiller (2010) qui déplore certaines utilisations erronées qui restent restreintes dans les limites des nations ou des identités ethniques:

Even scholars of transnational migration or diaspora have often bound their unit of study along the lines of national or ethnic identities. They have generally failed to link their descriptions of migrant local and trans-border connections 
to analyses of new flexible modes of capital accumulation and the contemporary neo-liberal restructuring of space, self and modes of social legitimation (Glick-Schiller 2010:111).

Dans mon travail de thèse, le terme " transnational " est mobilisé dans l'analyse de la pratique de la capoeira en dehors du Brésil, pour rendre compte de l'agency des individus, c'est-à-dire leur capacité d'agir et de prendre des décisions, à l'échelle individuelle mais aussi des organisations auxquelles ils adhèrent, appelées groupes ou associations de capoeira. Ces organisations présentent des rapports de pouvoir internes très complexes tout comme les rapports envers les autres groupes de capoeiristas qui configurent le marché de la capoeira sur place. Ces groupes sont formés par des individus aux origines les plus diverses, souvent transmigrants eux-aussi, qui retrouvent dans les groupes de capoeira un sens d'appartenance à des groupes ou des communautés (Bauman 2001). Les formes associatives des groupes de capoeira offrent une certaine sécurité et des repères à leurs membres dans des métropoles comme Londres et Paris.

Ainsi, à l'instar des études sur la transnationalisation des religions afro-américaines, dans le cas de la capoeira il s'avère nécessaire d'employer un concept de transnationalisation qui prenne en compte le fait que ce ne sont pas seulement les populations migrantes qui sont concernées par les processus de transnationalisation (Capone 2004, 2010). Si les Brésiliens ont un rôle important dans l'expansion de la capoeira en Europe, l'existence de groupes sous la direction des pratiquants locaux et leur conséquente appropriation de la pratique est de plus en plus notable, car elle se configure comme une tendance importante de ce processus d'expansion. Les réseaux complexes tissés entre les capoeiristas sur place et ceux du pays d'origine montrent qu'il est possible de faire partie des réseaux transnationaux sans nécessairement sortir de chez soi, mais en utilisant les nouvelles technologies, les échanges virtuels sur internet ou par un échange sur la pratique dans le pays d'origine (Capone 2010: 239).

\section{LES TROIS MOMENTS DE LA TRANSNATIONALISATION DE LA CAPOEIRA}

L'expansion de la capoeira hors du Brésil, malgré les histoires singulières de chaque parcours individuel des capoeiristas, peut être caractérisée par trois moments distincts du processus de transnationalisation de la pratique: premièrement, la circulation des capoeiristas et mestres brésiliens, associée par la suite à l'émigration des Brésiliens en quête de meilleures conditions de vie hors du Brésil; deuxièmement, I'installation des Brésiliens sur place dans divers pays; troisièmement, l'appropriation de la capoeira par des pratiquants locaux. Ces trois moments montrent que le processus de transnationalisation, dans le cas de la capoeira, est lui-même en constante reconfiguration et que la liaison à un lieu et l'identification à un groupe ou mestre de capoeira s'impose comme centrale dans le contrôle de l'expansion des groupes. Cependant, cette classification n'est pas restreinte à une périodicité strictement déterminée. En effet, les trois moments de la transnationalisation de la capoeira, identifiés ici, cherchent à mettre en évidence des spécificités qui ne sont pas strictement divisées temporellement.

Le premier moment de ce processus est incarné par le capoeirista brésilien qui se lance à l'étranger pour des spectacles folkloriques (Assunção 2005: 186-189) et qui par la suite décide de résider dans les pays du Nord en 
quête de meilleures conditions de vie et de travail. Cette phase peut être associée à la période de la fin des années 1970 jusqu'à la fin des années 1990. C'est pendant les années 1980 et 1990 que, en raison de la crise économique au Brésil, de nombreux Brésiliens ont émigré à la recherche de meilleures conditions de vie et de travail hors du Brésil. De cette façon, plusieurs individus, dont certains accompagnants de groupes folkloriques, ont découvert dans la capoeira, plus qu'une source de revenus, un moyen d'insertion par le biais de la valorisation positive de l'identité du pays d'origine. C'est ainsi que, lors de cette première phase de l'expansion de la pratique, des personnes avec peu ou de faibles connaissances sur la capoeira se sont parfois proclamés professeurs de capoeira ${ }^{21}$.

Au cours de cette phase, les capoeiristas ont fréquemment dû apprendre ou perfectionner la pratique de la capoeira une fois sur place, leur principal capital étant leur nationalité brésilienne, la maîtrise de la langue portugaise et une connaissance basique de la capoeira. D'autre part, le faible degré d'information disponible à l'époque sur la capoeira permettait qu'un pratiquant débutant au Brésil puisse, une fois à l'étranger, facilement affirmer sa légitimité face à d'autres concurrents sur ce marché, en raison de sa nationalité et du faible contrôle exercé par ses pairs.

L'autre caractéristique de cette première phase du processus de transnationalisation est le contrôle des nouveaux arrivés par les autres concurrents. Cette caractéristique a commencé à se développer alors que la pratique entamait les premiers pas de son expansion en dehors du Brésil. Lorsque la capoeira a commencé à réunir de nombreux groupes et enseignants dans des métropoles comme Paris et Londres, il a été de plus en plus difficile pour des " aventuriers " inconnus de se faire une place sur ce marché, vu le contrôle exercé par les capoeiristas eux-mêmes. La présence des mestres sur place et leur circulation, l'influence des nouvelles technologies comme l'accès à internet et aux vidéos de capoeira, les voyages au Brésil et les relations intergroupes ont considérablement accru le niveau de connaissance et de maîtrise de la capoeira pratiquée hors du Brésil, rendant plus difficile la nomination des " aventuriers ", n'ayant pas ou peu de connaissances, en tant que leaders de groupes. Si à ce moment le contrôle des pairs commence à s'exercer, il faut signaler que pendant cette période initiale, il existe l'envie de découvrir la pratique. Ainsi les capoeiristas sur place se rencontrent pour faire des rodas ensemble et coopèrent lorsqu'un autre pratiquant qui fait partie du réseau des capoeiristas arrive sur place.

Cela nous conduit à la deuxième phase du processus de transnationalisation de la capoeira, liée elle-aussi à l'immigration des Brésiliens. La différence de cette deuxième vague d'expansion de la capoeira réside dans le fait qu'il s'agisse de pratiquants de capoeira déjà plus expérimentés, ces nouveaux arrivants étant désormais reliés à des groupes ou des mestres au Brésil qui valident leur pratique en leur offrant un support et de la légitimité pour poursuivre leur activité face aux autres groupes présents sur le marché. Dans la plupart des cas, les capoeiristas de cette deuxième vague sont conscients de la réussite d'autres capoeiristas sur place. Une fois installés dans le pays d'accueil, ils découvrent que la capoeira peut devenir un métier qui va par la suite les obliger à se professionnaliser. La pratique de ces mestres et de leurs groupes se caractérise par une action corporative: une fois établis avec leurs groupes, ils font venir du Brésil leurs mestres et d'autres capoeiristas de la même lignée pour donner des stages et, lorsque cela est possible, créer d'autres groupes. De cette façon ils gagnent du capital symbolique au Brésil, puisqu'ils nourrissent le lien avec les capoeiristas de leur groupe d'origine. De plus, ils sont davantage valorisés et 
acquièrent plus de visibilité face aux autres concurrents sur le « marché local des biens culturels ». Les alliances et la coopération entre les groupes existent, mais il faut aussi remarquer les divisions entre les lignées et les écoles qui se reproduisent du Brésil sur le plan transnational.

Ce moment du processus de transnationalisation de la capoeira est marqué par une quête de la " tradition " et par l'affirmation de l'authenticité des groupes qui espèrent ainsi réunir plus d'élèves. D'un autre côté, ce moment est aussi marqué par la remise en cause de la légitimité des pratiquants qui ne possèdent pas de groupe ou de mestre, ainsi que celle des "mestres ou capoeiristas do avião ".

La deuxième phase va impulser les efforts de traduction culturelle de la part du capoeirista brésilien, dans l'objectif de comprendre les enjeux et les demandes locales pour adapter sa pratique. De cette capacité de traduction, de sa créativité à opérer une adaptation compréhensible pour les pratiquants locaux, va dépendre sa réussite ou son échec sur ce marché. Pour cela, il lui faut posséder des compétences personnelles pour identifier les demandes et mettre en place les transformations nécessaires à son insertion. Cela va entraîner inexorablement des ruptures avec la forme dont la capoeira est pratiquée au Brésil. Les capoeiristas ont besoin de réévaluer leurs exigences par rapport à l'engagement de l'élève, du port de l'uniforme jusqu'à la soumission aux caprices d'un mestre. Ils auront surtout besoin de se "professionnaliser ", comme dans le cas de certains groupes où la préoccupation de transformer la capoeira en un produit mieux adapté aux pratiquants locaux impose de réels changements. Dans cette deuxième phase, les capoeiristas brésiliens se voient dans la nécessité de négocier avec les besoins du marché local et d'imposer une plus grande rigueur à leur pratique, par exemple, par rapport au respect des horaires, à l'organisation et à la régularité des cours. S'établir et avoir des plans durables sur place peut être vu comme un atout du point de vue des élèves européens. D'autres qualités, comme la maîtrise de la langue locale, sont perçues comme importantes pour l'insertion.

Par conséquent, la troisième phase de l'expansion de la capoeira hors du Brésil est celle du capoeirista " local " qui s'approprie la capoeira et l'adapte à sa façon, opérant une véritable " traduction " de la capoeira au contexte local. La relocalisation de la pratique pendant cette troisième phase trouve exemple dans le cas de Mestre Fantasma du groupe East London Capoeira, du formado Carcará de l'association Kolors de France et de celui de Nicolas, ancien membre du groupe Ypiranga de Pastinha ${ }^{22}$. Ces derniers ne sont pas des Brésiliens, mais ils pratiquent et enseignent la capoeira depuis plus de dix ans à Londres et à Paris. Ces pratiquants et leurs groupes entretiennent des liens étroits avec le Brésil et y sont restés pendant des séjours d'une durée variable. Ils ont été autorisés à enseigner la capoeira et leurs groupes respectifs ont été validés par leurs mestres brésiliens.

L'appropriation par le pratiquant local se déroule en deux étapes. Dans un premier temps, le non-Brésilien s'associe à un mestre de capoeira et se soumet à ses règles et à celles d'un groupe pour être un légitime légataire de la pratique. Du point de vue du mestre brésilien, avoir un groupe en Europe signifie gagner plus de visibilité et de pouvoir pour faire face à ses concurrents du Brésil. L'association entre le mestre brésilien et son élève nonbrésilien va s'établir sur un rapport de confiance basé sur l'équilibre entre les exigences du mestre brésilien et ce que le groupe peut lui offrir, comme par exemple la réalisation d'un travail conjoint. Dans cet échange, le mestre lui 
accorde I'utilisation de son nom ou celui de son groupe et en contrepartie, le pratiquant local organise des stages et des visites du mestre et de son groupe en Europe. En revanche, si jamais les exigences du mestre deviennent trop lourdes et que les membres du groupe ne les acceptent plus en mettant ainsi en cause la légitimité de sa domination charismatique, le groupe peut alors ouvertement contester l'autorité du mestre, parfois en se séparant même de lui, comme ce fut le cas pour l'association Ypiranga de France. Cependant, cette séparation d'un mestre implique d'envisager la possibilité de se relier à un autre groupe ou à un autre mestre concurrent, dans le but de préserver le caractère légitime du groupe et de ses membres.

Ce troisième moment de la transnationalisation de la capoeira en Europe est relié à l'existence du mestre qui transite entre l'Europe et le Brésil, ou qui est basé durablement sur place. Avoir un mestre au Brésil, qui reconnaît et autorise l'existence du groupe, offre la possibilité de pratiquer la capoeira de forme légitime. La légitimité peut être également acquise si le mestre brésilien se trouve en Europe, comme cela a été le cas de l'association française Kolors reliée à Mestre Beija-Flor qui vivait à l'époque en France.

Ce troisième moment se caractérise par le besoin de légitimation des pratiquants et des groupes qui exercent leurs activités en Europe. Du point de vue du mestre du Brésil, les avantages de ce type de stratégie sont l'augmentation du pouvoir symbolique du mestre de capoeira face à ses concurrents locaux au Brésil, qui eux ne font pas partie de la circulation transnationale. Le mestre de capoeira cherche à maximiser ses profits en termes d'argent et de pouvoir symbolique issus de sa circulation.

Les trois phases de la transnationalisation de la capoeira mettent en évidence ce qui est au cœur de ce processus, à savoir la légitimité du pratiquant face à ses concurrents. Dans chaque moment, le rôle du mestre et sa liaison avec des pratiquants considérés comme légitimes sont des éléments indispensables à la relocalisation de la pratique hors du Brésil. II reste à signaler que le contrôle des mestres sert avant tout à identifier les pratiquants légitimes de la capoeira et par conséquent à renforcer de manière réciproque leur pouvoir, ce qui n'empêche pas forcément que d'autres groupes se créent en marge de ce réseau et qu'ils se proclament tout aussi légitimes que les autres.

\section{CONSIDERATIONS FINALES}

Relier le mouvement d'expansion de la capoeira hors du Brésil seulement à l'immigration des Brésiliens en quête de meilleures conditions de vie et de travail signifie oblitérer l'importance et la participation des agents locaux dans l'origine et la propagation des flux. Les non-Brésiliens sont des acteurs clés dans la création et le renforcement des réseaux transnationaux de capoeiristas, fréquemment ce sont eux-mêmes qui sont partis en quête d'apprendre la capoeira et ensuite former leurs groupes en liaison avec des mestres au Brésil.

Cependant, leurs lectures de la pratique ne sont pas non plus uniformes, elles expriment leurs histoires de vie, leurs stratégies d'affirmation dans ce marché et la vision des groupes ou des mestres auxquels ils sont affiliés. Alors de nouveaux lieux vecteurs de la " tradition " se créent dans les groupes hors du Brésil, passage obligatoire pour des pratiquants de capoeira à la recherche des racines de leurs mestres. Ces villes s'ajoutent à d'autres comme 
Salvador et Rio de Janeiro en tant qu'endroits clés producteurs de significat pour les processus de construction de l'identité collective de ces groupes et individuelle des pratiquants. La multiplication des lieux de " tradition " de la capoeira au Brésil est donc conséquence de la circulation des capoeiristas. Ces derniers profitent du prestige acquis à l'étranger pour apporter des ressources traduites par l'envoi d'argent ou d'élèves dans leurs villes et groupes d'origines. Encore une fois, c'est par le biais des idées disséminées au sein des groupes de capoeira que cette valorisation est rendue possible.

Au cœur de ce débat, la tension entre " tradition " et changement est présente de manière forte dans ce processus de transnationalisation. Les conséquences de la transnationalisation de la capoeira sont elles aussi ressenties par les pratiquant non-brésiliens. Ainsi, comme le signale Capone (2004:11), ce n'est pas seulement le migrant qui est concerné par le processus de transnationalisation, dans le cas de la capoeira, les pratiquants nonbrésiliens sont affectés par la transnationalisation de la pratique et créent ou découvrent des nouvelles formes d'appropriation de la pratique " afro-brésilienne ». 


\section{NOTAS}

1 Cet article a été élaboré à partir d'une thèse de doctorat en Ethnologie et Histoire "Les mestres, les groupes et les "lieux dynamiques" Identité et relocalisation de la pratique de la capoeira à Paris et à Londres " dans le cadre d'une cotutelle entre l'Université de Paris Ouest Nanterre La Défense et l'University of Essex, sous la direction de Stefania Capone et Matthias Röhrig Assunção. Ce travail soutenu en 2013 a été rendu possible grâce à une bourse de cotutelle de thèse accordée par l'Université de Paris Ouest Nanterre La Défense et à une bourse de la Région Ile de France, dispositif SETCI (Soutien à l'encadrement de thèse en cotutelle internationale).

2 Les batucadas sont des orchestres musicaux composés par des instruments de percussion, des tambours de taille variable qui jouent des rythmes " afro-Brésiliens". La samba est un type de musique aux origines " afro-brésiliennes " très apprécié au Brésil. La "brésilité " ou l'" afro-brésilité " sont des manifestations issues des pratiques populaires brésiliennes qui sont devenues des symboles du pays et qui sont identifiées comme des pratiques du Brésil à l'étranger. Ces pratiques se sont diffusées principalement à travers les spectacles folkloriques, et par l'initiative des Brésiliens qui décident de rester à l'étranger et d'offrir des cours de ces modalités. Le cas de la capoeira est analysé par Assunção (2005: 185-189) qui remarque l'importance des spectacles folkloriques dans la diffusion de la capoeira aux États-Unis et en Europe.

3 Un article de Sarah Barrell publié par The Independent le 01/07/2001 intitulé "Capoeira The mysterious South American martial art now has devotees all over the UK" souligne la présence de deux capoeiristas brésiliens parmi tous les élèves d'origines diverses du cours auquel elle avait assisté au London School of Capoeira.

4 L'absence d'un sentiment d'appartenance communautaire entre les migrants brésiliens aux États-Unis est expliquée par Margolis (2003: 59) par le fait que les Brésiliens vont aux États-Unis pour travailler et gagner un maximum d'argent sur une courte période. Comme ils cumulent deux ou trois emplois, ils n'ont pas le temps de rejoindre des organisations communautaires. Les Brésiliens, d'après l'auteur, renient leur condition d'immigré et affirment qu'ils sont aux États-Unis ponctuellement, dans l'intention de rentrer dès que possible dans leur pays.

5 La recherche effectuée dans le cadre de ma thèse s'est concentrée sur deux groupes en France: I'association Kolors de Paris et le groupe Ypiranga de Pastinha d'Ivry-sur-Seine. Au Royaume-Uni, la recherche a été ciblée sur les activités de cinq groupes londoniens: London School of Capoeira, Amazonas, East London Capoeira, Cordão de Ouro Londres et Kabula.

6 Certaines de ces études, comme celle de Delamont et Stephens (2008: 61), reconnaissent l'importance de la migration des Brésiliens pour comprendre la capoeira hors du Brésil, sans toutefois analyser la bibliographie sur les Brésiliens migrants ni les spécificités de cette migration. D'autres comme celle de Joseph (2008a: 197-199) sur la capoeira au Canada, et de Guizardi (2011: 365-414) à propos de la capoeira à Madrid, remarquent l'importance de l'immigration des Brésiliens pour l'explication de la croissance de la capoeira dans leur pays respectif. La thèse d'Aceti reconnait le rôle de l'immigration des pratiquants brésiliens mais met en évidence la participation et l'importance de pratiquants non-brésiliens en Europe (Aceti 2011: 234-236).

7 Par rapport à la capoeira à Madrid, l'étude de Guizardi (2011) signale que parmi les vingt et un groupes qu'elle a étudiés à Madrid, onze ont été créés au Brésil et se sont implantés à Madrid grâce à la présence sur place de Brésiliens, et dix ont été créés à Madrid, six d'entre eux étant dirigés par des Brésiliens migrants et quatre par des capoeiristas européens. Elle remarque que près d'un tiers des groupes de Madrid composant son échantillon (de 2007 à 2009) est dirigé par des Européens pour la plupart espagnols et portugais (Guizardi 2011: 30).

8 Il est plus précis d'utiliser le terme « non-brésilien » pour désigner les capoeiristas aux origines les plus diverses à Londres et à Paris. Ainsi, lorsqu'on parle de capoeiristas londoniens ou parisiens, il s'agit de ceux qui pratiquent la capoeira sur place, sans qu'ils soient nécessairement originaires de ces deux villes.

9 En France, les termes "globalisation " et " mondialisation " sont employés de manière interchangeable (cf. Abélès 2008). Au long de la thèse, j'ai choisi d'utiliser le terme " globalisation ".

10 Falcão $(2006,2008)$ utilise la notion d'internationalisation pour décrire le processus d'expansion de la capoeira à partir des années 1970. Il signale l'importance des médias dans la diffusion de la pratique (Falcão 2006: 66).

11 Dans un article présenté en 2009 à I'University of Essex, j'ai analysé le processus d'appropriation d'images de la capoeira en France et au Royaume-Uni par le biais de publicités télévisées, diffusées dans ce pays, ainsi que la forme dont ces publicités s'approprient la capoeira et la traduisent dans des versions locales (Ferreira 2009). 
12 Le sens de la notion de diaspora a beaucoup changé comme le remarque Bordes-Benayoun et Schnapper qui rappellent que « dans l'imaginaire de notre époque, il y aurait des exils heureux, qui procurent un sentiment de liberté et de plaisir de découvrir de nouveaux univers et de celui résister à la fatalité d'un destin tout tracé » (2006: 14).

13 Pour expliquer l'usage contemporain du terme diaspora et les changements avec l'usage classique, Faist (2010) signale que les usages contemporains ne distinguent pas les raisons de la migration, si elle est forcée ou volontaire, et que l'accent sur le retour a été remplacé par des échanges circulatoires ou par la mobilité transnationale et finalement, la coexistence entre l'intégration et de la distinction culturelle des populations concernées (Faist 2010: 13).

14 Cohen (1997: 26) à partir d'un modèle idéal typique weberien dénombre neuf caractéristiques communes aux différentes diasporas, bien que, d'après l'auteur, aucune diaspora ne s'identifie exactement au modèle proposé. Même si l'expérience des Brésiliens, capoeiristas ou non, peut trouver sa place dans certaines des caractéristiques proposées, les Brésiliens, et encore moins les capoeiristas, ne s'auto-identifient pas en tant que " peuple diasporique ».

15 Une étude récente met en évidence l'importance des élèves locaux de Mestre João Grande dans l'installation, le support et la permanence du mestre à New York (cf. Castro 2007).

16 Certes, la participation et l'importance des Brésiliens dans la diffusion de la capoeira hors du Brésil est encore remarquable, mais nous constatons une tendance à l'appropriation de la pratique par les pratiquants locaux, qui négocient de plus en plus leur place au sein de ce marché. La difficulté à comprendre l'expansion de la capoeira par une diaspora de Brésiliens fait l'objet de réflexion pour Aceti (2011: 235-236) qui signale l'importance des pratiquants non brésiliens pour l'expansion de la pratique de la capoeira en Europe.

17 L'alliance avec les locaux est notable car quand les groupes sont dirigés par des Brésiliens, il est nécessaire de compter sur les élèves qui jouent le rôle de véritables traducteurs et interprètes pour le contexte local. II n'est pas rare que les Brésiliens capoeiristas se trouvent, à l'intérieur des groupes, des compagnons qui vont avoir une place importante dans l'organisation du groupe, en y exerçant un rôle de secrétariat et fréquemment en hébergeant le capoeirista et en s'acquittant de ses factures, comme l'a expliqué Aceti (2011: 441).

18 La notion d' "invention de la tradition » développée par Hobsbawm dans l'introduction du volume organisé par Hobsbawm et Ranger (1983) est ici applicable parce qu'elle met en évidence le caractère construit de ces connexions. D'après l'auteur le processus d'invention des traditions consiste essentiellement en un processus de formalisation et ritualisation, imposé par la répétition et qui est caractérisé par la référence au passé (Hobsbawm 1983: 4). Cependant, la notion de tradition dans les "cultures des diasporas", proposée par Gilroy (1993) et Clifford (1997) nous semblent offrir une approche plus précise pour cette étude.

19 Trad.: "Des identifications, et non des identités, des actes de relation plutôt que des formes pré-établies: cette tradition est un réseau d'histoires partiellement connectées, un espace/temps constamment déplacé et réinventé ».

20 Assunção (2005) a été l'un des premiers à avoir mis en lumière l'ampleur du phénomène de l'expansion de la capoeira hors du Brésil et les enjeux du contact entre les Brésiliens et les non-Brésiliens. Son travail est important dans la constitution d'un champ d'étude sur la capoeira hors du Brésil. Ce sujet est traité aussi de manière profonde et systématique par d'autres auteurs comme Delamont (2006) Delamont et Stephens (2007, 2008) Delamont et al. (2010) à propos de la capoeira et des groupes au Royaume-Uni. Ces auteurs ont mis en place des formes d'écriture ethnographiques innovantes sur la capoeira. De même, pour l'étude sur un groupe de capoeira à Lyon, Brito (2011) s'inspire de la notion de "glocal » de Robertson (1992). Dans son long travail de thèse sur l'expansion de la capoeira en Europe, Aceti (2011) analyse des sujets concernant les tensions entre le local et le global, ainsi que les enjeux de la déterritorialisation et de la relocalisation de la capoeira en Europe (Aceti 2011: 238-9).

21 La critique récurrente formulée par les mestres de capoeira à l'encontre de ces pratiquants est traduite par l'expression " mestre do avião " (mestre de l'avion) qui fait référence aux Brésiliens qui ne sont pas enseignants de capoeira au Brésil mais qui, une fois débarqués de l'avion, se métamorphosent en professeurs ou en "mestres".

22 Formado est une catégorie indigène du groupe Senzala de Santos duquel Carcará (surnom de capoeira de Jocelyn Chaubo) fait partie; ce terme désigne dans son groupe celui qui est prêt à dispenser des cours de capoeira. Ce titre est attribué par le mestre, dans le cas de Jocelyn, Mestre Beija-Flor. Les parcours de ces capoeiristas et leurs groupes est analysé em détail dans ma thèse de doctorat (Ferreira 2013). 


\section{REFERÊNCIAS BIBLIOGRÁFICAS}

ABÉLÈS, Marc. 2008. '« Politique et globalisation » Perspectives anthropologiques'. L'Homme 1(185/186): 133-143.

ACETI, Monica. 2011. Devenir et rester capoeiriste en Europe. Transmissions interculturelles et mondialité de la capoeira Afro-Brésilienne. PhD Dissertation. Université de Franche Comté.

ASSUNÇÃO, Matthias Röhrig. 2005. Capoeira: The History of an Afro-Brazilian Martial Art. London: Routledge.

BASCH, Linda; GLICK SCHILLER, Nina; SZANTON BLANC, Cristina. 1994. Nations Unbound: Transnational Projects, Postcolonial Predicaments and the Deterritorialized Nation-State. New York: Gordon and Breach.

BORDES-BENAYOUN, Chantal e SCHNAPPER, Dominique. 2006. Diasporas et Nations. Paris: Odile Jacob.

BRITO, Celso. 2011. Capoeira Angola "glocalizada": análise etnográfica de um grupo da cidade de Lyon, França. In Reunião de Antropologia do Mercosul 11. Anais... Curitiba: PPGAS-UFPR.

BRUNEAU, Michel. 2010. "Diasporas, Transnational Spaces and Communities". In: T. Faist \& R. Bauböck (orgs.). Diaspora and Transnationalism. Amsterdam: Amsterdam University Press.

CAPONE, Stefania. 2004. "À propos des notions de globalisation et de transnationalisation". Civilisations 51(1/2): 9-22.

2010. "Religions « en migration »: De l'étude des migrations internationales à l'approche transnationale". Autrepart 4(56):235-259.

CASTRO, Mauricio Barros. 2007. Na Roda do Mundo: Mestre João Grande entre a Bahia e Nova York. 2007. Ph.D Dissertation. São Paulo: Universidade de São Paulo.

CHIVALLON, Christine. 2002. "La diaspora noire des Amériques : Réflexions sur le modèle de l'hybridité de Paul Gilroy". L'Homme 161: 51-73.

CHIVALLON, Christine e BERTHOMIÈRE, William (orgs.). 2006. Les diasporas dans le monde contemporain. Paris: Karthala. CLIFFORD, James. 1994. "Diasporas". Cultural Anthropology 9 (3): 302-338.

Press.

1997. Routes: Travel and translation in the late twentieth century. Cambridge, Massachusett: Harvard University

COHEN, Robin. 1997. Global Diasporas: An Introduction. Seattle: University of Washington Press.

2006. "'Diaspora' changing meanings and limits of the concept". In: C. Chivallon \& W. Berthomière (orgs.). Les diasporas dans le monde contemporain. Paris: Karthala.

DELAMONT, Sara. 2006. "The Smell of Sweat and Rum: Authority and Authenticity in Capoeira Classes". Ethnography and Education 1: 161-176.

DELAMONT, Sara e STEPHENS, Neil. 2007. Excruciating Elegance: Representing the Embodied Habitus of Capoeira. ESRC National Centre for Research Methods NCRM - Working Paper Series: 1-37.

DELAMONT, Sara et STEPHENS, Neil. 2008. "Up on the roof: the embodied habitus of diasporic capoeira". Cultural Sociology 2(1): 57-74.

DELAMONT, Sara; CAMPOS, Claudio e STEPHENS, Neil. 2010. "I'm your teacher! I'm Brazilian!" Sport, Education and Society 15(1): 103-120. 
FAIST, Thomas e BAUBÖCK, Rainer (orgs.). 2010. Diaspora and Transnationalism. Amsterdam: Amsterdam University Press. FALCÃO, Jose Luiz Cirqueira. 2006. "O Jogo da Capoeira em Jogo". Revista Brasileira de Ciência do Esporte 27(2): 59-74. 2008. "A Internacionalização da Capoeira". Revista textos do Brasil (1): 123-133.

GRANADA, Daniel. 2004. Brasileiros nos Estados Unidos: capoeira e identidades transnacionais. MA Thesis. Rio de Janeiro: Universidade Federal do Rio de Janeiro.

2005. La capoeira du Brésil à Paris. MA Thesis. Paris: IHEAL Institut des Hautes Études de l'Amérique Latine, Université de Paris III Sorbonne Nouvelle.

2007. A capoeira do Brasil até Paris: redes sociais, transformações e adaptações da prática da capoeira no Brasil e na França. In: CEISAL V Congreso Europeo Ceisal. Anais... Bruxelas: CEISAL. Disponível em: <http://www.reseauamerique-latine.fr/ceisal-bruxelles/MS-MIG/MS-MIG-2-Granada.pdf> Acesso em: 27 mar. 2012. (24): $63-86$

2008. "Adaptação em Movimento: O processo de 'transnacionalização' da capoeira na França". Revista Antropolítica

2009. Contemporary capoeira narratives in commercial advertisements in France and the United Kingdom. Comunicação oral apresentada no evento Capoeira from Regional to Global, University of Essex, Colchester.

(20):39-43

2010. "'Fazer o bem, faz bem': Notas sobre identidade e o aprendizado da capoeira na França". Revista da Fundarte

2015. Pratique de la capoeira em France et au Royaume Uni. Paris: Harmattan.

GILROY, Paul. 1993. The Black Atlantic: modernity and Double Consciousness. London: Verso.

GLICK SCHILLER, Nina ; BASCH, Linda ; SZANTON BLANC, Cristina. 1995. "From Immigrant to Transmigrant: Theorizing Transnational Migration". Anthropological Quarterly 68(1): 48-63.

GLICK SCHILLER, Nina ; LEVITT, Peggy. 2004. "Conceptualizing Simultaneity: A Transnational Social Field Perspective on Society". International Migration Review 38 (3): 1002-1039.

GLICK-SCHILLER, Nina. 2010. A global perspective on transnational migration: Theorising migration without methodological nationalism. In: T. Faist \& R. Bauböck (orgs.). Diaspora and Transnationalism. Amsterdam: Amsterdam University Press: 109-130.

GRAVINA, Heloisa. 2010. Por cima do mar eu vim, por cima do mar eu vou voltar. Ph.D Dissertation. Porto Alegre, RS: Universidade Federal do Rio Grande do Sul.

GUIZARDI, Menara Lube. 2011. Todo lo que la boca come. Flujos, rupturas y fricciones de la capoeira en Madrid. Ph.D Dissertation. Madrid: Universidad Autonoma de Madrid.

HALL, Stuart. 1990. "Cultural Identity and Diaspora". In: J. Rutherford (org). Identity: Community, Culture and Difference. London: Lawrance and Wishart.

HANNERZ, Ulf. 1996. Transnational Connections. London: Routledge.

HOBSBAWN, Eric. 1983. "Introduction: Inventing Traditions". In: T. Ranger \& E.Hobsbawn (orgs.). The invention of traditions. Cambridge: Cambridge University Press.

JOSEPH, Janelle. 2008a. "Going to Brazil': transnational and corporeal movements of a Canadian-Brazilian martial arts 
community". Global Networks 8(2):194-213.

2008b. "The Logical Paradox of the Cultural Comodity: Selling an 'Authentic' Afro-Brazilian Martial Art in Canada". Sociology of Sport Journal 25 (a): 498-515.

MARGOLIS, Maxine. 1994. Little Brazil: Imigrantes brasileiros em Nova York. Campinas: Papirus.

2003. "Na virada do milênio a imigração brasileira para os Estados Unidos". In: A. C. B. Martes \& S. Fleischer (orgs.). Fronteiras Cruzadas. São Paulo: Paz e Terra.

MARTES, Ana Cristina Braga. 1999. Brasileiros nos Estados Unidos - um estudo sobre imigrantes em Massachusetts. São Paulo: Paz e Terra.

2003. "Raça e etnicidade - Opções e Constrangimentos". In: A. C. B. Martes \& S. Fleischer (orgs.). Fronteiras Cruzadas. São Paulo: Paz e Terra.

PATARRA, Neide Lopes. 2005. "Migrações internacionais de e para o Brasil contemporâneo". São Paulo em Perspectiva 19(3): 23-33.

PENHA, Marcelo Montes 2001. "African Heritage and National Representation: Two cases of Brasilidade in New York City". Raízes e Rumos 7: 372-388.

REIS, Rossana Rocha e SALES, Teresa (orgs.). 1999. Cenas de um Brasil migrante. São Paulo: Boitempo.

RIBEIRO, Gustavo Lins. 1999. "’O que faz o Brasil, Brazil: Jogos identitários em São Francisco'". Cenas do Brasil Migrante. São Paulo: Boitempo: 45-85.

ROBERTSON, Roland. 1992. Globalization - Social Theory and Global Culture. London: Sage.

SALES, Teresa. 1999. Brasileiros longe de casa. São Paulo: Cortez.

2005. "Eles vestem o avental da América". O Estado de São Paulo 20 mar. 2005 (Caderno Aliás): J 4-5.

TRAVASSOS, Sonia Duarte. 2000. Capoeira, difusão e metamorfoses culturais entre Brasil e Estados Unidos. 2000. Ph.D Dissertation. Rio de Janeiro: Museu Nacional, Universidade Federal do Rio de Janeiro.

VASSALLO, Simone Ponde. 2001. Ethnicité, tradition et pouvoir : le jeu de la capoeira à Rio de Janeiro et à Paris. Ph.D Dissertation. Paris: EHESS École des Hautes Études en Sciences Sociales.

WESOLOWSKI, Katya. 2012. "Professionalizing Capoeira: Politics of Play in Twenty-first-Century Brazil". Latin American Perspectives 39(2): 82-89. 
Echanges globaux, agents locaux. I'apport de la capoeira aux études sur la mondialisation

RÉSUMÉ

L'article analyse le processus d'expansion de la capoeira, un art martial d'origine « afro-brésilienne ", hors du Brésil. À partir du débat sur l'internationalisation de la pratique nous problématisons l'apport des concepts d'immigration, globalisation, diaspora et transnationalisation pour comprendre ce phénomène. En se basant sur la recherche de terrain réalisée en France et au Royaume-Uni entre 2007 et 2011, la compréhension du processus d'expansion de la capoeira à l'étranger est interprétée en trois moments distincts mais reliés et qui ne sont pas restreints à une temporalité stricte. L'importance des pratiquants locaux non-Brésiliens est mise en évidence, aussi bien à l'origine que dans l'entretien du mouvement de l'expansion de la capoeira em Europe.

MOTS-CLÉS: Capoeira; transnationalisation; mondialisation; identité.

\section{Trocas globais, agentes locais. a contribuição da capoeira aos estudos sobre mundialização}

\section{RESUMO}

O artigo analisa o processo de expansão da capoeira, uma arte marcial "afro-brasileira", fora do Brasil. A partir do debate sobre a internacionalização da prática buscamos mostrar a contribuição dos conceitos de imigração, globalização, transnacionalização e diáspora para compreender este fenômeno. O trabalho é baseado em uma pesquisa de capoeira realizada na França e no Reino Unido entre 2007 e 2011. São identificados três momentos distintos e inter-relacionados da expansão da prática da capoeira no exterior. São colocados em relevo a importância dos praticantes locais, não-brasileiros tanto na origem quanto na manutenção do movimento de expansão da capoeira na Europa.

PALAVRAS-CHAVE: Capoeira; transnacionalização; mundialização; identidade 